ORIGINAL ARTICLE

\title{
Comprehensive isokinetic knee measurements and quadriceps tendon evaluations in footballers for assessing functional performance
}

\author{
L Özçakar, B Kunduracyoolu, A Cetin, B Ülkar, R Guner, Z Hascelik
}

Br J Sports Med 2003;37:507-510

See end of article for authors' affiliations

Correspondence to: Dr Özçakar, Hacettepe University Medical School, Physical Medicine and Rehabilitation, Ankara 06100, Turkey;

lozcakar@yahoo.com

Accepted 16 June 2003

\begin{abstract}
Objectives: To ascertain whether detailed isokinetic knee muscle testing reflects the results of other functional measurements in footballers and to look for any correlations between quadriceps tendon thickness and knee strength.

Methods: Ultrasonographic evaluation of the quadriceps tendon (Hitachi EUB-405), isokinetic knee testing (Biodex System 3), and sprint measurements using telemetric photoelectric cells (Chronometre Prosport ESC TX02) were carried out on 29 elite footballers. Jumping capacity was evaluated using Bosco's jumping mat (Ergojump). Anaerobic fitness was assessed by auricular capillary blood lactate measurements (YSI Model 1500 Sport Lactate Analyzer).

Results: Quadriceps tendon thickness correlated positively with jumping and sprint measurements and negatively with extensor and flexor strength. However, these correlations did not reach statistical significance. There were significant correlations between knee extensor strength at $60 \%$ and jumping or sprint measurements and between the extension acceleration values of both knees during isokinetic tests at $240 \%$ and the sprint measurements. No significant correlation was found between the fatigue ratio values of both knees at $240 \% \mathrm{~s}$ and the calculated fatigue ratios from the sprint measurements.

Conclusions: Apart from a few variables which correlated with the performance tests, the isokinetic studies did not fully predict the various functional measurements. Neither was there any relation between the quadriceps tendon measurements and the knee strength values nor with the functional performance.
\end{abstract}

sokinetic muscle testing is often used to evaluate strength in sports medicine. A wide variety of objective and reproducible data can readily be obtained ${ }^{12}$ from convenient measurements. When promptly interpreted, these data can be used to assess functional performance.

Several studies have compared the results of isokinetic knee testing with certain functional measurements in footballers, who require various skills of different intensities to play their sport..$^{3-7}$

In this study, we aimed to ascertain whether the results of isokinetic muscle testing would reflect the results of other functional measurements in elite footballers. More precisely, we wanted to correlate several variables of isokinetic testing for predicting data acquired from the other types of measurement. The comparative evaluations for which we sought correlations were: quadriceps tendon (QT) thickness and strength, sprint, or jumping measurements; peak torque values and sprint or jumping measurements; extension acceleration times and sprint measurements; fatigue ratios during isokinetic measurements with the ratios calculated from the sprint tests or lactate analysis.

\section{MATERIALS AND METHODS}

The subjects were 29 elite footballers (from a team in the first division) aged 18-31. Each player was given detailed information on the whole procedure before testing began.

Ultrasonographic evaluation of the quadriceps tendon During the measurements, the players sat on the edge of the examination table with their legs hanging freely and their knees at $90^{\circ}$ of flexion. QT thickness was measured with a $7.5 \mathrm{MHz}$ linear probe (Hitachi EUB-405) in longitudinal section with the probe parallel to the tendon's alignment, near its insertion in the patella. Any accompanying pathologies were noted.

\section{Isokinetic muscle testing}

Isokinetic muscle measurements were performed using the Biodex System 3 (Biodex Medical Systems, Inc, New York, New York, USA) dynamometer with the knee attachment on. Orientation of the dynamometer was kept at $0^{\circ}$, tilt at $0^{\circ}$, seat orientation at $0^{\circ}$. Before the testing procedure, all the players performed conditioning exercises and stretching of the lower extremities to warm up. They then exercised on a treadmill for 10 minutes. They were seated and secured to the apparatus with straps across the chest and thigh. Each time, the attachments of the dynamometer were readjusted accordingly, so that the centre of motion of the lever arm was aligned as accurately as possible with the slightly changing flexion-extension axis of the joint. The resistance pad was placed on the distal tibia. The range of motion of the knee joint was set at $0-90^{\circ}$. Bilateral isokinetic (concentric/ concentric) knee flexion and extension studies within the protocol of $60 \%$ (five repetitions), $240 \%$ (15 repetitions) were accomplished. Between the two sessions, players rested for 20 seconds. Vocal encouragement during the tests was consistent and standardised. Flexion and extension peak torque values, acceleration times, and fatigue ratios, automatically calculated by the device, were noted.

\section{Functional performance tests}

Sprint measurements were carried out by using telemetric photoelectric cells placed at 0,5, 10, and $20 \mathrm{~m}$ (Chronometre

Abbreviations: QT, quadriceps tendon 
Table 1 Basic details and quadriceps tendon (QT) thickness measurements of the footballers studied

\begin{tabular}{lll}
\hline & Range & Mean (SD) \\
\hline Age (years) & $18.00-31.00$ & $23.62(3.57)$ \\
Body weight (kg) & $64.30-105.40$ & $76.12(8.34)$ \\
Height (m) & $1.70-1.92$ & $1.81(0.05)$ \\
QT (right) (mm) & $5.80-9.10$ & $6.84(0.58)$ \\
QT (left) (mm) & $5.60-10.10$ & $6.83(0.75)$ \\
\hline
\end{tabular}

Prosport ESC TX02, Tümer Engineering, Ankara, Turkey). The players stood $1 \mathrm{~m}$ behind the starting line, started on a verbal signal, and then ran as fast as they could to complete the $20 \mathrm{~m}$ distance. They repeated the test 10 times with 30 second rest periods in between. The fastest of 10 trials and fatigue ratios comparing the first with the last trial and the first three with the last three trials were used for subsequent analysis.

Jumping capacity was evaluated using Bosco's jumping mat (Ergojump; Globus Italia, Codogne, Italy). The players performed a squat jump and a counter movement jump with the arms kept akimbo to eliminate any contribution from them. The best result of three tests was recorded for further analysis.

Anaerobic fitness was assessed while the players ran on a treadmill, with a starting velocity of $10 \mathrm{~km} / \mathrm{h}$ for three minutes. Then the speed was increased to $12 \mathrm{~km} / \mathrm{h}$ and by $1 \mathrm{~km} / \mathrm{h}$ onwards every three minutes until exhaustion. Blood lactate concentrations of $6 \mathrm{mM}$ were reached. Auricular capillary blood lactate was measured, every three minutes, with a lactate analyser (YSI Model 1500 Sport Lactate Analyzer; Yellow Springs Instrument Co, Yellow Springs, Ohio, USA). Running speed and heart rate at 2, 2.5, 3, 4, and $6 \mathrm{mM}$ lactate were all recorded, and those at 2 and $4 \mathrm{mM}$ lactate were included in the statistical analysis.

Statistical Package for Social Sciences (SPSS) 10.0 for windows was used throughout the statistical analysis, and Pearson correlation coefficients were primarily taken into consideration.

\section{RESULTS}

Table 1 summarises the descriptive anthropometric data and the QT thickness measurements. The QT measurements on both sides correlated strongly $(r=0.90, \mathrm{p}=0.00)$, whereas body weight correlated weakly only with QT thickness on the right side $(r=0.39, \mathrm{p}=0.05)$.

Tables 2 and 3 show the isokinetic knee evaluation and functional test results respectively of the players. The peak
Table 2 Isokinetic measurements of the knee joints

\begin{tabular}{|c|c|c|}
\hline & Range & Mean (SD) \\
\hline PT Ext $60 \%$ s (right) (N.m) & $282.8-490.7$ & 361.58 (47.09) \\
\hline PT Ext $60 \%$ (left) (N.m) & $267.7-446.3$ & $366.59(41.96)$ \\
\hline PT Flex $60 \%$ s (right) (N.m) & $113.5-241.9$ & $187.78(32.13)$ \\
\hline PT Flex $60 \%$ (left) (N.m) & $122.4-242.1$ & $179.28(26.36)$ \\
\hline PT Ext $240 \%$ s (right) (N.m) & $140.3-247.1$ & $194.90(30.38)$ \\
\hline PT Ext $240 \%$ s (left) (N.m) & $142.9-256.4$ & 200.79 (27.89) \\
\hline PT Flex $240 \%$ s (right) (N.m) & $105.8-176.7$ & $137.48(19.63)$ \\
\hline PT Flex $240 \%$ s (left) (N.m) & $92.8-169.7$ & $124.36(18.50)$ \\
\hline FR Ext $240 \%$ s (right) (\%) & $-0.1-46.5$ & $31.17(10.30)$ \\
\hline FR Ext $240 \%$ (left) (\%) & $10.2-44.4$ & $31.24(8.62)$ \\
\hline FR Flex $240 \%$ s (right) (\%) & $4.5-59.8$ & $36.70(11.56)$ \\
\hline FR Flex $240 \%$ s (left) (\%) & $18.7-71.5$ & $42.36(11.42)$ \\
\hline Acc Ext $240 \%$ s (right) (ms) & $30.0-80.0$ & $47.50(12.66)$ \\
\hline Acc Ext $240 \%$ s (left) (ms) & $30.0-70.0$ & $51.43(10.79)$ \\
\hline Acc Flex $240 \% \mathrm{~s}$ (right) (ms) & $40.0-100.0$ & 61.43 (15.33) \\
\hline Acc Flex $240 \%$ s (left) (ms) & $40.0-100.0$ & 69.29 (14.38) \\
\hline
\end{tabular}

PT, Peak torque; Flex, flexion; Ext, extension; FR, fatigue ratio; Acc, acceleration.

Table 3 Functional test results

\begin{tabular}{lll}
\hline & Range & Mean (SD) \\
\hline SJ $(\mathrm{cm})$ & $34.10-49.10$ & $41.75(4.31)$ \\
$\mathrm{CMJ}(\mathrm{cm})$ & $38.50-56.50$ & $44.80(4.51)$ \\
$5 \mathrm{~m}(\mathrm{~s})$ & $0.76-1.03$ & $0.91(0.07)$ \\
$10 \mathrm{~m}(\mathrm{~s})$ & $1.51-1.83$ & $1.69(0.07)$ \\
$20 \mathrm{~m}(\mathrm{~s})$ & $2.64-3.07$ & $2.86(0.12)$ \\
Lac 2 vel (m/s) & $2.78-4.25$ & $3.42(0.39)$ \\
Lac 2 HR (/s) & $124.00-176.00$ & $155.52(12.22)$ \\
Lac 4 vel (m/s) & $3.33-4.75$ & $4.12(0.32)$ \\
Lac 4 HR (/s) & $160.00-191.00$ & $176.61(7.47)$ \\
\hline \multicolumn{3}{l}{ SJ, Squat jump; CMJ, counter movement jump; Lac, lactate; vel, velocity; } \\
HR, heart rate.
\end{tabular}

torque values of knee extensors and flexors measured at $60 \%$ and $240 \%$ velocities correlated intrinsically (all $\mathrm{p}<0.05$ ). Among the functional performance test results, there were positive intrinsic correlations in each group: squat jump and counter movement jump values; 5, 10, and $20 \mathrm{~m}$ passing times; heart rate and running speed during lactate analysis (all $\mathrm{p}<0.05$ ).

QT thickness, on both the right and left side separately, correlated positively with the two types of jumping and sprint measurements, and negatively with the extensor and flexor peak torque values. None of these correlations reached significance (all $\mathrm{p}>0.05$ ).

Table 4 Correlations between isokinetic, jumping, and sprint measurements

\begin{tabular}{llllllll}
\hline & PT (R) & PT (L) & SJ & CMJ & $5 \mathbf{~ m}$ & $10 \mathbf{~ m}$ & $20 \mathbf{~ m}$ \\
\hline PT (R) & $(-)$ & 0.68 & 0.41 & 0.33 & -0.20 & -0.40 & -0.44 \\
& & $(0.00)$ & $(0.03)$ & $(0.09)$ & $(0.31)$ & $(0.04)$ & $(0.02)$ \\
PT (L) & 0.68 & $(-)$ & 0.40 & 0.55 & -0.44 & -0.52 & -0.58 \\
& $(0.00)$ & & $(0.03)$ & $(0.00)$ & $(0.02)$ & $(0.00)$ & $(0.00)$ \\
SJ & 0.41 & 0.40 & $(-)$ & 0.66 & -0.43 & -0.56 & -0.58 \\
& $(0.03)$ & $(0.03)$ & & $(0.00)$ & $(0.02)$ & $(0.00)$ & $(0.00)$ \\
CMJ & 0.33 & 0.55 & 0.66 & $(-)$ & -0.37 & -0.41 & -0.53 \\
& $(0.09)$ & $(0.00)$ & $(0.00)$ & & $(0.05)$ & $(0.03)$ & $(0.00)$ \\
$5 \mathrm{~m}$ & -0.20 & -0.44 & -0.43 & -0.37 & $(-)$ & 0.84 & 0.79 \\
& $(0.31)$ & $(0.02)$ & $(0.02)$ & $(0.05)$ & & $(0.00)$ & $(0.00)$ \\
$10 \mathrm{~m}$ & -0.40 & -0.52 & -0.56 & -0.41 & 0.84 & $(-)$ & 0.90 \\
& $(0.04)$ & $(0.00)$ & $(0.00)$ & $(0.03)$ & $(0.00)$ & & $(0.00)$ \\
$20 \mathrm{~m}$ & -0.44 & -0.58 & -0.58 & -0.53 & 0.79 & 0.90 & $(-)$ \\
& $(0.02)$ & $(0.00)$ & $(0.00)$ & $(0.00)$ & $(0.00)$ & $(0.00)$ & \\
\hline
\end{tabular}

Values are $r$ values with $\mathrm{p}$ values in parentheses. Values in bold are significant.

PT, Peak torque; SJ, squat jump; CMJ, counter movement jump. 
There were significant correlations among extensor peak torque values at $60 \%$, jumping, and sprint measurements (table 4). On the other hand, the same type of correlations between the flexor peak torque values at $60 \%$ were not significant.

Values for extension acceleration time of both knees, right and left respectively, during isokinetic measurements at $240 \%$ correlated positively with the $10 \mathrm{~m}$ passing times $(r=0.37, \mathrm{p}=0.05 ; r=0.38, \mathrm{p}=0.05)$ and for the left knee; these values also correlated with the $20 \mathrm{~m}$ passing times $(r=0.43, \mathrm{p}=0.02)$.

Fatigue ratios for each knee at $240 \%$, during extension and flexion, correlated with those of the other knee (all $\mathrm{p} \leqslant 0.001)$; however, when compared with the calculated fatigue ratios from the sprint measurements (first versus last trial and first three versus last three trials), no significant correlation was found. Nor was there any correlation with the variables obtained from lactate analysis-heart rate and running speed (all $\mathrm{p}>0.05$ ).

\section{DISCUSSION}

One of the most important variables for measuring performance in team sports is physical condition. In football, however, because of the complexity of the game, it is difficult to ascertain the contribution of this variable to the functional outcome. Talent and technical and tactical strategies are the other contributing factors. We mainly focused on lower extremity muscular strength and anatomical features to explain the relative contributions to functional performance, which was evaluated by commonly used tests. We did not attempt to relate the results of any test to playing position but it is known that the endurance demands for the more active midfield players are higher than for defenders and forwards, who often display higher levels of vertical jump and sprinting performance. $^{89}$ Neither did we try to relate the measurements to playing level (elite versus subelite or amateur) as we conducted our study on a single professional team comprising elite players. It is known that such measurements vary according to the player's level, ${ }^{4}$ elite players usually having stronger knee flexor muscles than amateurs.

In football, the quadriceps muscle is crucial in running, jumping, and kicking, and the hamstrings control running and stabilize the knee joint, ${ }^{10}$ which becomes even more important with increased speed. ${ }^{11}$ Our study yielded similar results, as the quadriceps muscle strength of the players correlated with the sprint and jumping measurements (table 4). As expected, as the players' strength increased, their passing times decreased and their jumping values increased. We noticed that the correlations were a little more significant for the left side measurements but we have no explanation for this. This is in accordance with a study by Saliba and Hrysomallis, ${ }^{12}$ who evaluated the relation between lower limb strength and jumping and kicking performance. They found low to moderately significant correlations between isokinetic measures and vertical jumping and inferred that strengthening the knee musculature may enhance jumping performance. In contrast, Cometti et al, ${ }^{4}$ reporting no correlations between maximal strength and anaerobic power performance, pointed out the necessity of determining the most relevant protocol for footballers. There are also studies on healthy subjects that have reported moderate to highly significant correlations between the isokinetic testing of the knee extensors and some functional performance tests such as one leg hop, ${ }^{13-15}$ vertical jump, ${ }^{16-19}$ standing long jump, and five step jump. ${ }^{16}$ On the other hand, the lack of correlation between the knee extensor strength and certain functional tests, such as square hop, one leg rising, draws attention to the contribution of other factors such as skill, endurance, coordination, and postural control. ${ }^{3}$
Take home message

Isokinetic studies along with functional measurement tests are valuable tools for assessing the performance of footballers. We believe that both are necessary and one cannot replace the other. On the other hand, we emphasise that soft tissue ultrasonography is gaining merit for biomechanical evaluation, rather than being simply a diagnostic tool.

Besides the isokinetic knee strength, we also looked at the acceleration times and fatigue ratios of the players during isokinetic measurements. To the best of our knowledge, neither the comparison of isokinetic acceleration values with sprint measurements nor comparison of the two fatigue ratios (isokinetic $v$ sprint measurements) have been done before. We thought that isokinetic acceleration values may reflect sprint performances. The $10 \mathrm{~m}$ passing times correlated with the measurements on both sides, and $20 \mathrm{~m}$ passing times with measurements on the left side. Similarly, we looked for a correlation between the fatigue ratios of isokinetic and sprint measurements. We were unable to show any significant correlation, nor with the lactate analysis. This implies that isokinetic studies alone will not suffice to reflect the other test results as far as fatigue and acceleration are concerned.

In our study, QT thickness on both sides was evaluated by ultrasonography. Our aim was to relate the sonographic variables with either the strength measurements or the performance tests. Although they correlated to a certain extent, the significance was low. It has been shown that, as the tendon transmits larger forces, the calcified tissue at its insertion increase ${ }^{20}$; thus it may be more useful to measure this calcification with ultrasound to obtain an idea of muscle strength. However, QT thickness was found to correlate with body weight $(p=0.05)$.

Overall, we suggest that isokinetic knee strength evaluations in footballers, and their prompt interpretation, may give an idea about their physical conditioning. They may reflect some results of related functional tests and be beneficial in the follow up of a rehabilitation programme, but cannot replace other specific functional performance measurements. We think that more relevant studies should be carried out to explain the relations-between the isokinetic tests results and the other types of measurement - that we have highlighted. It is reasonable to suggest that ultrasonographic evaluation of the force transmitting anatomical structures will-other than simply being useful for examining injuries-have merit in speculating on biomechanical properties.

\section{Authors' affiliations}

L Özçakar, A Cetin, Z Hascelik, Hacettepe University Medical School, Physical Medicine and Rehabilitation, Ankara, Turkey

B Kunduracyoolu, B Ülkar, R Guner, Department of Sports Medicine, Ankara University Medical School, Ankara, Turkey

\section{REFERENCES}

1 Kannus $\mathbf{P}$, Jarvinen M. Prediction of torque accleration energy and power of thigh muscles from peak torque. Med Sci Sports Exerc 1989;21:304-7.

2 Tegner $Y$, Lysholm J, Lysholm M, et al. A performance test to monitor rehabilitation and evaluate anterior cruciate ligament injuries. Am J Sports Med 1986; 14:156-9.

3 Östenberg A, Roos E, Ekdahl C, et al. Isokinetic knee extensor strength and functional performance in healthy female soccer players. Scand J Med Sci Sports 1998:8:257-64.

4 Cometti G, Maffiuletti NA, Pousson M, et al. Isokinetic strength and anaerobic power of elite, subelite and amateur French soccer players. Int J Sports Med 2001;22:45-51.

5 Quarrie KL, Wilson BD. Force production in the rugby union scrum. J Sports Sci 2000;18:237-46. 
6 Orchard J, Marsden J, Lord S, et al. Preseason hamstring muscle weakness associated with hamstring muscle injury in Australian footballers. Am J Sports Med 1997;25:81-5.

7 Östenberg A, Roos H. Injury risk factors in female European football. A prospective study of 123 players during one season. Scand J Med Sci Sports 2000; 10:279-85

8 Davis JA, Brewer J, Atkin D. Pre-season physiological characteristics of English first and second division soccer players. J Sports $\mathrm{Sc}_{\mathrm{i}}$ 1992; 10:541-7.

9 Wisloff U, Helgerud J, Hoff J. Strength and endurance of elite soccer players. Med Sci Sports Exerc 1998;30:462-7.

10 Fried T, Lloyd GJ. An overview of common soccer injuries. Management and prevention. Sports Med 1992;14:269-75.

11 Hagood S, Solomonow M, Baratta R, et al. The effect of joint velocity on the contribution of the antagonist musculature to knee stiffness and laxity. Am J Sports Med 1990;18:182-7.

12 Saliba L, Hrysomallis C. Isokinetic strength related to jumping but not kicking performance of Australian footballers. J Sci Med Sport 2001;4:336-47.

13 Tegner Y, Lysholm J, Lysholm M, et al. A performance test to monitor rehabilitation and evaluate anterior cruciate ligament injuries. Am J Sports Med 1986;14:156-9.
14 Greenberger HB, Paterno MV. Relationship of knee extensor strength and hopping test performance in the assessment of lower extremity function. J Orthop Sports Phys Ther 1995;22:202-6.

15 Barber SD, Noyes FR, Mangine RE, et al. Quantitative assessment of functional limitations in normal and anterior cruciate ligament-deficient knees. Clin Orthop 1990;225:204-14.

16 Wiklander J, Lysholm J. Simple tests for surveying muscle strength and muscular stiffness in sportsmen. Int J Sports Med 1987;8:50-4.

17 Anderson MA, Gieck JH, Perrin D, et al. The relationships among isometric, isotonic, and isokinetic concentric and eccentric quadriceps and hamstring force and three components of athletic performance. J Orthop Sports Phys Ther 1991;14:114-20.

18 Oddsson LIE, Westing SH. Jumping height can be accurately predicted from selected measurements of muscle strength and biomechanical parameters. Biomechanics in Sports 1991;9:29-33.

19 Lassau V, Pocholle M, Bernard PL, et al. Evaluation de la puissance maximale des membres inferieurs. A partir d'un test de terrain et de tests isocinetiques. Annales de Kinesitherapie 1995;22:193-202.

20 Evans EJ, Benjamin M, Pemberton DJ. Variations in the amount of calcified tissue at the attachments of the quadriceps tendon and patellar ligament in man. J Anat 1991;174:145-51.

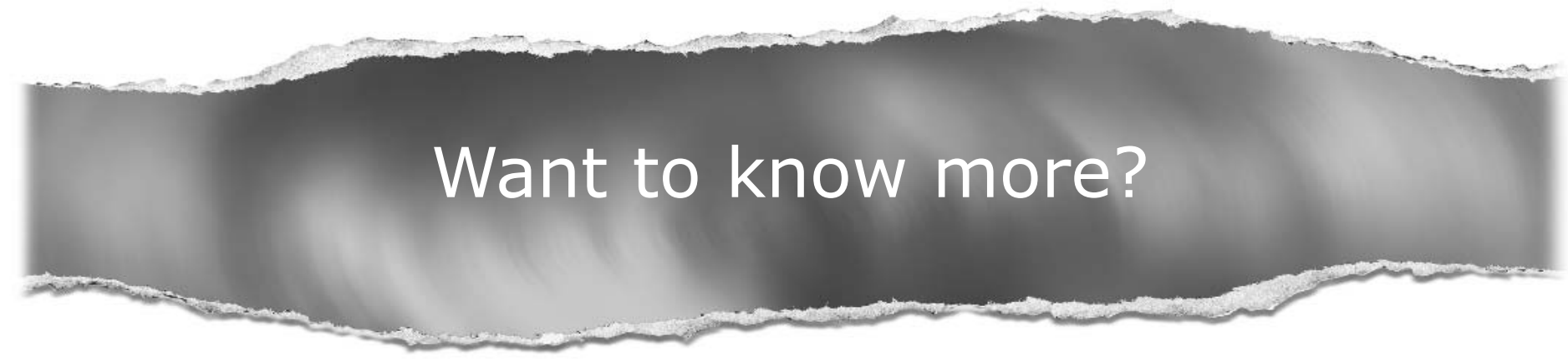

Data supplements

Limited space in printed journals means that interesting data and other material are often edited out of articles; however, limitless cyberspace means that we can include this information online.

Look out for additional tables, references, illustrations.

www.bjsportmed.com 